\title{
Diffuse Alveolar Hemorrhage: Wegener’s Granulomatosis Relapse after Inadequate Treatment
}

\author{
GE Carpagnano*, D Lacedonia, A Koutelou, E Costantino and MP Foschino-Barbaro
}

Institute of Respiratory Disease, Department of Medical and Occupational Sciences, University of Foggia, Italy

\begin{abstract}
Granulomatosis with Polyangiitis (GPA) represent the most common Antineutrophil Cytoplasmic Autoantibody (ANCA)-associated vasculitis. The treatment modalities indicated for the initial manifestations are steroids and cyclophosphamide therapy. Diffuse alveolar hemorrhage is a life- threatening though rare complication of GPA. A case of GPA with diffuse alveolar hemorrhage and hypoxemic respiratory failure is reported, as the manifestation of the disease, after inadequate drug regimen and that responded to aggressive cyclophosphamide and steroids therapy and non invasive mechanical ventilation.
\end{abstract}

\section{Introduction}

Granulomatosis with polyangiitis (GPA) also note as Wegener's granulomatosis (WG) is a necrotizing vasculitis that affects small and medium-size blood vessels with granulomata formation [1]. It is one of the most common forms of systemic vasculitis [1]. The causes of the disease are unknown, and it is the prototype of conditions associated with anti-neutrophilic cytoplasmic antibody (ANCA) [2]. It affects mainly middle-aged Caucasian individuals, without gender predilection [3]. The clinical presentation can vary, and it may affect several organs. The most common symptoms are related to the upper and lower airways, especially recurring bloody rhinorrhea, rhinosinusitis, and cavitary and nodular lesions in the lungs, along with glomerulonephritis. The diagnosis is based on clinical manifestations, histopathological findings compatible with granulomatous necrotizing vasculitis [1] and the presence of ANCA. Cytoplasmic ANCA (c-ANCA) pattern has specificity for GPA of up to $98 \%$ in the acute phase [4]. To induce remission of the active form of GPA, prednisolone (40 to $60 \mathrm{mg} /$ day) and cyclophosphamide ( 50 to $100 \mathrm{mg} /$ day) are administered orally for 8 to 12 weeks. After induction of remission, patients receive maintenance therapy, prednisolone plus cyclophosphamide or prednisolone only without cyclophosphamide. Azathioprine is also recommended for maintenance therapy.

Un uncommon complication of the GPA is the Diffuse Alveolar Hemorrhage (DAH). DAH can be defined by the presence of hemoptysis, diffuse alveolar infiltrates, and a drop in hematocrit level [5]. Symptoms include cough, hemoptysis, dyspnea, and anemia. However, chest radiographic and CT findings are nonspecific (the alveolar infiltrates can even sometimes be unilateral), and hemoptysis may be lacking [6]. The diagnosis is usually made with bronchoscopy, by means of which serial bronchoalveolar lavage samples (from the same location) reveal an increasing red blood cell count. For the treatment of diffuse alveolar damage-induced DAH, high-dose intravenous methylprednisolone end cyclophosphamides are recommended.

\section{Case Report}

In 2011, a 25-year-old Ukrainian white man presented with a 2-year history of fever, cough and asthenia. His symptoms had begun in April 2009. The patient initially received a pulmonary TB diagnosis and an antituberculous drug regimen was prescribed. After almost two years, because of otalgia, persistent high fever, arthralgias, myalgias, nasal fullness and blood-stained sputum, he was admitted to a Ukraine Hospital. GPA diagnosis was made during that admission and a course of corticosteroids (prednisolone) was then prescribed. Despite corticosteroid treatment, his otalgia, low-grade fever and arthralgias continued.

On April 11, 2011, he was admitted to our hospital with complaints for dyspnea, productive cough, and intermittent episodes of hemoptysis, otalgia, nasal fullness and low-grade fever. Since his last hospitalization, he continued the oral coticosteroid regimen. There was no past history of any allergic diathesis. He was a driver by occupation and non-smoker.

On general examination, he was pale and weak. His resting pulse rate was $116 / \mathrm{min}$, blood pressure $115 / 65 \mathrm{mmHg}$, and respiratory rate $24 / \mathrm{min}$. He was also febrile. Chest examination was unremarkable on inspection. On palpation, vocal tactile fremitus was reduced in the middle and lower lung fields. On percussion a dull sound was produced on the left lung fields. On auscultation, rhonchi were audible on the left lung fields, but was otherwise clear. His hearing had decreased bilaterally.

Initial laboratory studies included as follows: $\mathrm{Hb}-9.7 \mathrm{~g} \%$, TLC $13430 /$ ul, DLC - neutrophils - 90.4\%, lymphocytes - 3.8\%, monocytes $-4.8 \%$, platelet count $-478,000 /$ ul, erythrocyte sedimentation rate -80 , serum bilirubin $-0.6 \mathrm{mg} / \mathrm{dl}$, SGPT - $73 \mathrm{IU}$, blood urea $-30 \mathrm{mg} / \mathrm{dl}$, and serum creatinine $-0.7 \mathrm{mg} / \mathrm{dl}$. Arterial blood gas analysis, in room air, done at the time of admission proved no hypoxia with $\mathrm{PaO} 286 \mathrm{mmHg}$ and $\mathrm{PaCO} 236 \mathrm{mmHg}$. Urinalysis indicated red blood cells in absence of proteins. Birmingham Vasculitis Activity Score (BVSA):22. Because of the falling hematocrit level ( $\mathrm{Hb} 7,2$; Hct 21\%), blood transfusion was required.

The chest radiograph revealed bilateral parenchymal consolidation in the middle lung fields (Figure 1a).

*Corresponding author: Dr. Giovanna Elisiana Carpagnano, Institute of Respiratory Disease, Department of Medical and Occupational Sciences, University of Foggia Italy, Tel: 00390881733143; Fax:00390881733158; E-mail: ge.carpagnano@unifg.it

Received June 07, 2012; Accepted July 18, 2012; Published July 20, 2012

Citation: Carpagnano GE, Lacedonia D, Koutelou A, Costantino E, FoschinoBarbaro MP (2012) Diffuse Alveolar Hemorrhage: Wegener's Granulomatosis Relapse after Inadequate Treatment. J Clin Case Rep 2:177. doi:10.4172/2165 7920.1000177

Copyright: (C) 2012 Carpagnano GE, et al. This is an open-access article distributed under the terms of the Creative Commons Attribution License, which permits unrestricted use, distribution, and reproduction in any medium, provided the original author and source are credited. 
On CT scan, alveolar consolidation with air bronchogram, appeared in conjuction with typical cavitating lesions, in the posterior middle lung fields, without hilar lymphoadenopathy (Figure 1b).

The autoimmune work-up was entirely negative except from c-ANCA.

A bronchoscopy was performed and Bronchoalveolar lavage (BAL) samples came out to be hemorrhagic consistent with alveolar hemorrhage.

BAL cultures were negative for bacterial infection while the cytology did not detect any malignant cells.

Sputum cultures for acid-fast bacilli and bacteria were negative. Nasal and throat swabs for influenza virus H1N1 were negative.

The antiviral serologic profile, revealed positivity on HCV antibodies.

Despite broad-spectrum antibiotic and corticosteroid moderate dose regimen, during his hospitalization, dyspnea was rapidly progressive with hemoptysis and drop in hematocrit level was persistent. Arterial blood gas analysis in room air done, revealed hypoxia with $\mathrm{PaO}_{2} 52 \mathrm{mmHg}$ and $\mathrm{PaCO}_{2} 39 \mathrm{mmHg}$. Blood gas abnormalities became rapidly so severe that non invasive mechanical ventilation for hypoxemic respiratory failure was necessary. For about seven days red blood cells were found in Urinalysis ( $\max 165 \mathrm{Rbc} / \mathrm{L}$ ). Intravenous therapy with methylprednisolone, initially up to $1 \mathrm{~g}$ daily in divided doses and intravenous cyclophosphamide $50 \mathrm{mg}$ bid, was started. Daily dose of methylprednisolone was reduced after three days at $40 \mathrm{mg}$ bid. Four units of blood were transfused to correct anemia. A new CT scan was performed after 8 days since the aggressive immunosuppressive therapy was started (Figure 2). A radiographic improvement was revealed, with diffuse ground-glass opacities, representing alveolar involvement, in conjuction with cavitating lesions in the posterior upper and middle lung fields.

Rapid clinical improvement was obtained with the complete resolution of the hypoxic respiratory failure. Ear, Nose and Throat Clinic and Nephrology Clinic were consulted for further evaluation. Pre- existing fibrotic changes were observed on the tympanic membrane and blood-stained mucus was found at the nasal cavities. Renal biopsy showed periglomerular leukocytes infiltrated, interstitial edema, occasional rupture of Bowman's capsule and fibrinoid necrosis of the glomerular.

The patient was discharged asymptomatic (BVAS=2 and any significant altered values at blood analysis) and maintenance immunosuppressive therapy with cyclophosphamide (100 mg/die) was prescribed.

One year later, the patient is alive and well. Follow-up chest radiograph shows improvement of the bilateral middle lung field's consolidation (Figure 3).

\section{Discussion}

Diffuse Alveolar Hemorrhage (DAH) is caused by diffuse intraalveolar bleeding from the small vessels of the lungs, particularly the alveolar capillaries, but also the arterioles and venules. The many different entities causing DAH may be classified into three groups: (a) ANCA-associated small-vessel vasculitis, which generally involves pulmonary capillaritis (GPA, CSS, microscopic polyangiitis); (b)
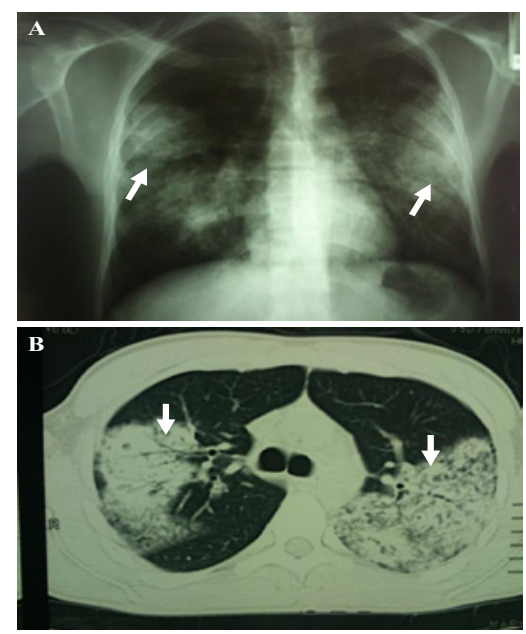

Figure 1: A: chest radiograph of patients at the beginning; B: CT scan of patients at the beginning (white arrows show the consolidations).

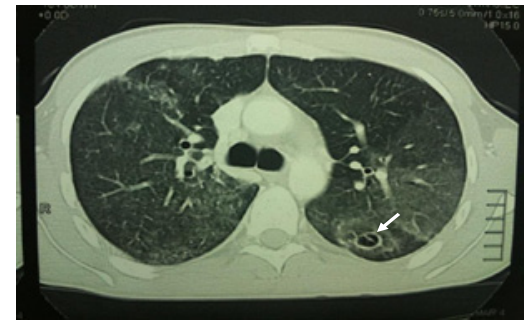

Figure 2: CT scan of patients after 8 days since the aggressive immunosppressive therapy (white arrow shows excavated lesion in right upper lobe).

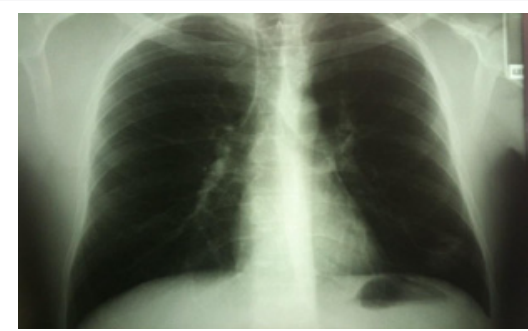

Figure 3: Follow-up chest radiograph.

syndromes caused by immune deposits that can be detected with immunofluorescence, such as Good pasture syndrome and systemic lupus erythematosus; and (c) a large group of miscellaneous entities that includes diffuse alveolar damage, drug reactions (including cocaine inhalation), coagulopathies, infections, and idiopathic disease such as idiopathic pulmonary hemosiderosis [7].

Most cases of DAH are caused by capillaritis associated with systemic autoimmune diseases such as ANCA-associated small-vessel vasculitis, Goodpasture syndrome, and systemic lupus erythematosus [8]. DAH occurs in approximately $10 \%$ of patients with Wegener granulomatosis [9].

Granulomatosis with polyangiitis is a disorder characterized by granulomatous inflammation and necrotizing vasculitis in various organs. The most common radiographic and CT abnormalities, seen at presentation in up $90 \%$ of patients, consist of lung nodules and masses [6]. Airspace consolidations and patchy or less commonly diffuse 
Citation: Carpagnano GE, Lacedonia D, Koutelou A, Costantino E, Foschino-Barbaro MP (2012) Diffuse Alveolar Hemorrhage: Wegener's Granulomatosis Relapse after Inadequate Treatment. J Clin Case Rep 2:177. doi:10.4172/2165-7920.1000177

ground-glass opacities are the second most common radiographic finding (20\%-50\% of cases) and may occur with or without associated lung nodules and masses. The consolidations and ground-glass opacities reflect either vasculitic pulmonary disease in the form of pneumonitis or alveolar hemorrhage. Hemoptysis is the hallmark of DAH, but as many as $33 \%$ of patients are seen without hemoptysis [7]. Cough, progressive dyspnea, fatigue and fever may accompany the hemoptysis. When DAH is extensive, respiratory failure may occur.

Immunosuppressive agents are the mainstay of therapy for DAH. In those with life-threatened vital organ loss, initial therapy requires highdose cyclophosphamide and methylprednisolone for the induction of remission. Besides corticosteroids, other immunosuppressive drugs such as cyclophosphamide, azathioprine, mycophenolate mofetil, methotrexate, and etanercept may be used in diffuse alveolar hemorrhage, especially when the condition is severe, when first-line therapy with corticosteroids has proven ineffective or when a specific underlying cause is present.

While DAH is a manifestation of many diseases, GPA was confirmed by c-ANCA positivity, radiographic consolidation and hemorrhagic bronchoalveolar lavage. Infective diseases were rule out, because of viral and bacterial respiratory absence. Malignant disorders were excluded since cytology did not show any malignant cells.

This case emphasizes the importance of an aggressive immunosuppressive therapy in order to increase survival in patients affected by DAH in the setting of a systemic vasculitis such as Granulomatosis with polyangiitis.

\section{References}

1. Stone JH (2007) Wegener granulomatosis. In current rheumatology diagnosis \& treatment, 2nd ed. United States of America, Lange Medical Books/ McGrawHill.

2. Antunes T, Barbas CSV (2005) Granulomatose de Wegener. J Bras Pneumo 31: s21-26.

3. Klippel JH, Dieppe PA (1998) Rheumatology, v. 2, 2nd ed. United States of America. Mosby International.

4. Nölle B, Specks U, Lüdemann J, Rohrbach MS, DeRemee RA, et al. (1989) Anticytoplasmatic autoantibodies: their immunodiagnostic value in Wegener granulomatosis. Ann Intern Med 111: 28-40.

5. Primack SL, Miller RR, Müller NL (1995) Diffuse pulmonary hemorrhage: clinical, pathologic, and imaging features. AJR Am J Roentgenol 164: 295-300.

6. Marten K, Schnyder P, Schirg E, Prokop M, Rummeny EJ, et al. (2005) Patternbased differential diagnosis in pulmonary vasculitis using volumetric CT. AJR Am J Roentgenol 184: 720-733.

7. Green RJ, Ruoss SJ, Kraft SA, Duncan SR, Berry GJ, et al. (1996) Pulmonary capillaritis and alveolar hemorrhage. Update on diagnosis and management. Chest 110: 1305-1316.

8. Castañer E, Alguersuari A, Gallardo X, Andreu M, Pallardó Y, et al. (2010) When to Suspect Pulmonary Vasculitis: Radiologic and Clinical Clues. Radiographics 30: 33-53.

9. Franks TJ, Koss MN (2000) Pulmonary capillaritis. Curr Opin Pulm Med 6: 430-435. 